\title{
Influence of Finite Ground Conductivity on the Propagation of VLF Radio Waves
}

\author{
James R. Wait and Kenneth P. Spies \\ Central Radio Propagation Laboratory, National Bureau of Standards, Boulder, Colo.
}

(Received May 24, 1965)

\begin{abstract}
Calculations of the modal characteristics of the earth-ionosphere waveguide are presented for a wide range of ground conductivities. It is shown that the attenuation rate of the dominant modes, as a function of ground conductivity, has a maximum value which depends on frequency and various waveguide parameters. One of the important findings is that frequencies of the order of $10 \mathrm{kc} / \mathrm{s}$ may be more adversely affected by very-low conductivity than frequencies of the order of $30 \mathrm{kc} / \mathrm{s}$.
\end{abstract}

\section{Introduction}

In most numerical treatments of VLF propagation in the earth-ionosphere waveguide, it is assumed that the ground is perfectly conducting [e.g., Al'pert, 1960; Volland, 1961; Budden, 1962; Johler and Berry, 1962; Galejs, 1965]. While this is surely a reasonable assumption for propagation over sea water, it is certainly not valid for over-land paths [Wait, 1957].

It is the purpose of this paper to consider the influence of a finitely conducting ground on the characteristics of the waveguide modes in the VLF range. To encompass all cases of practical interest, a fairly wide range of ground conductivities is considered. However, in order to restrict the curves to a manageable number, a fixed ionospheric model is assumed although the reflecting height is allowed to vary somewhat.

\section{Description of the Model}

The earth and the ionosphere are assumed to be spherically concentric. The earth is smooth and homogeneous with conductivity $\sigma_{g}$ and dielectric constant $K$ (relative to free space). A convenient quantity to describe the characteristics of the lower ionosphere is the "conductivity parameter" $\omega_{r}$ which is defined by [Wait, 1962; Budden, 1962]

$$
\omega_{r}=\omega_{0}^{2} / \nu
$$

where $\omega_{0}$ is the angular plasma frequency of the electrons and $\nu$ is the effective collision frequency. A survey [Wait and Spies, 1964] of recent electron density profile data shows that a reasonable daytime model of the lower ionosphere is specified by the relation

$$
\omega_{r}=\left(2.5 \times 10^{5}\right) \exp [\beta(z-h)]
$$

where $\beta$ is a constant, $z$ is the height above the earth's surface and $h$ is a reference height. When $z$ and $h$ are measured in $\mathrm{km}$, it would appear that $\beta=0.3 \mathrm{~km}^{-1}$ is a representative value for the daytime ionosphere. At least, this is the case in the region where obliquely incident VLF radio waves are reflected. At nighttime, it is not to be expected that this exponential form is adequate; however, for present purposes, this model is retained.

When the effect of the earth's magnetic field must be considered, it is necessary to specify both the height profile of the plasma frequency $\omega_{0}$ and the collision frequency $\nu$. Assuming these are exponential functions, it follows that:

$$
\omega_{0}^{2}=\text { const } \times \exp (\beta-a) z,
$$

and

$$
\nu=\text { const } \times \exp (-a z),
$$

where $a$ is the inverse scale height for the collision frequency profile. A representative value for both daytime and nighttime conditions is $a=0.15 \mathrm{~km}^{-1}$ [Wait and Spies, 1964]. To display the effect of the earth's magnetic field in an ecomonical manner, the direction of propagation is chosen to be along the magnetic equator [Crombie, 1958]. The results are not quite as restrictive as they might first seem since, for arbitrary directions of propagation, it is known that the transverse component is most important for reflection of radio waves at oblique incidence [Wait, 1962].

A convenient magnetic field parameter is defined by

$$
\Omega= \pm \omega_{T} / \nu,
$$

where $\omega_{T}$ is the (angular) gyrofrequency of electrons in the earth's magnetic field. The quantity is negative for propagation from the WEST TOWARDS THE EAST, while it is positive for propagation from the EAST TOWARDS THE WEST.

For the model described above, the vertical electric field, $E$, at a great circle distance, $d$, from a ground- 
based vertical electric dipole source, is given by

$$
E=E_{0} W,
$$

where $E_{0}$ is a reference or inverse-distance field and $W$ is the propagation function. Following earlier conventions [Wait, 1962],

$$
W \cong \frac{(d / \lambda)^{1 / 2}}{(h / \lambda)} e^{-i \pi / 4} \sum_{n=1}^{\infty} \exp \left[i k d C_{n}^{2} / 2\right] G_{n} \Lambda_{n},
$$

where $\lambda$ is the wavelength, $k=2 \pi / \lambda, G_{n}$ is a heightgain function, $C_{n}$ is a complex coefficient, and $\Lambda_{n}$ is the excitation factor for mode $n$. The attenuation rate of the modes is $\operatorname{Im}\left(k C_{n}^{2} / 2\right)$ nepers per unit distance, while the phase velocity relative to the velocity of light is $1+\operatorname{Re} C_{n}^{2} / 2$.

The numerical methods used for obtaining the modal coefficients $C_{n}$ and the complex excitation factors $\Lambda_{n}$ have been discussed at some length in a recent technical note [Wait and Spies, 1964], so details need not be given here.

When the observer is on the surface of the earth, the height-gain function $G_{n}$ may be replaced by unity. When the receiving antenna is elevated one should refer to general expressions for the height-gain functions; however, for most cases of practical interest, the following expansion is satisfactory [Wait, 1962]:

$$
G_{n} \cong 1+i \Delta k z-\frac{C_{n}^{2}(k z)^{2}}{2}-\frac{1}{3}\left[\frac{1}{k a}+i \frac{C_{n}^{2} \Delta}{2}\right](k z)^{3},
$$

where $a$ is the radius of the earth, $z$ is the height of the observer above the earth's surface, and $\Delta$ is the normalized surface impedance of the earth's surface. In terms of the electrical characteristics of the ground

$$
\Delta=\left(\frac{i \epsilon_{0} \omega}{\sigma_{g}+i K \epsilon_{0} \omega}\right)^{1 / 2}\left(1-\frac{i \epsilon_{0} \omega}{\sigma_{g}+i K \epsilon_{0} \omega}\right)^{1 / 2},
$$

where $\omega$ is the angular frequency and $\epsilon_{0}$ is the permittivity of free space.

When $G_{n}$ is near unity (i.e., small heights), it is not difficult to show that

$$
\left|G_{n}\right| \cong 1-\frac{|\Delta| k z}{\sqrt{2}}-\left(\frac{v_{n}}{c}-1\right)(k z)^{2},
$$

where $v_{n} / c$ is the phase velocity of mode $n$ relative to the velocity of light. To within the same approximation,

$$
\text { phase of } G_{n} \cong \frac{|\Delta| k z}{\sqrt{2}}-\alpha_{n}(k z)^{2},
$$

where $\alpha_{n}=k \operatorname{Im} C_{n}^{2} / 2$ is the attenuation rate in nepers per unit length.

The height-gain functions are actually related to the excitation factor $\Lambda_{n}$. In fact, the following identity exists [Wait, 1962]:

$$
\Lambda_{n}=\frac{h / 2}{\int_{0}^{h}\left[G_{n}(z)\right]^{2} d z} .
$$

In the case of a flat and perfectly conducting earth, $G_{n}(z)$ may be replaced by $\cos \left(k C_{n} z\right)$. Then, if at the same time the upper boundary were a perfect reflector, it is easy to show that $\Lambda_{n}$ is unity. With this normalization for the excitation factor, it may well turn out that $\left|\Lambda_{n}\right|$ is sometimes greater than unity when the ground is finitely conducting. However, more than not, $\left|\Lambda_{n}\right|$ is somewhat less than unity when both the ground and the ionosphere are imperfectly reflecting.

\section{Discussion of Numerical Results}

Using the model of the earth-ionosphere waveguide as described above, extensive calculations have been carried out for the attenuation, phase velocity, and excitation factor of the modes. A fairly comprehensive description of the numerical methods employed is given in a published technical note [Wait, and Spies, 1964] which also contains extensive numerical data. Here, some of the results are presented in graphical form with particular emphasis on the effect of finite ground conductivity. A number of curves for perfect ground conductivity are also shown to facilitate comparison.

The attenuation rate (in decibels per $1000 \mathrm{~km}$ ) as a function of frequency is shown in figure 1 (for mode number 1) for the exponential ionosphere model and for a perfectly conducting ground. Various reference or reflection heights are shown and the magnetic field parameter $\Omega$ takes the values $-1,0$, and +1 .

It is evident from the curves in figure 1 that the attenuation rate for propagation from the west toward the east (i.e., $\Omega<0$ ) has the least attenuation. This is in conformity with much of the experimental data on the subject. An important feature of the curves in figure 1 is that the minimum attenuation for daytime heights (i.e., $h \sim 70 \mathrm{~km}$ ) is around $16 \mathrm{kc} / \mathrm{s}$ whereas at night (i.e., where $h \sim 90 \mathrm{~km}$ ), the minimum attenuation is around $12 \mathrm{kc} / \mathrm{s}$. These features are also quite consistent with the published experimental data [Obay ashi et al., 1959; Taylor, 1960; Watt and Croghan, 1964].

The magnitude of the excitation factor as a function of frequency is shown in figure 2 for the same conditions as in figure 1. The ordinate is expressed in decibels $(\mathrm{dB})$. It is certainly evident that the excitation factor decreases with increasing frequency. In particular, it is seen that for the greater reflecting heights, the excitation of mode $l$ is relatively weak. It is also interesting to note that the earth's magnetic field appears to have a very small effect on the excitation factor

The phase velocity as a function of frequency is shown in figure 3 which is for the same conditions as figure 1. These have the expected shape of dispersion curves for propagation in conventional waveguides; however, one should note that the phase velocity $v$ for mode 1 may be either greater or less than the velocity of light $c$. This interesting phenomenon is a result of the curved nature of the waveguide. It would appear, at least for the model employed, that the earth's magnetic field has an exceptionally small effect on the phase velocity. 


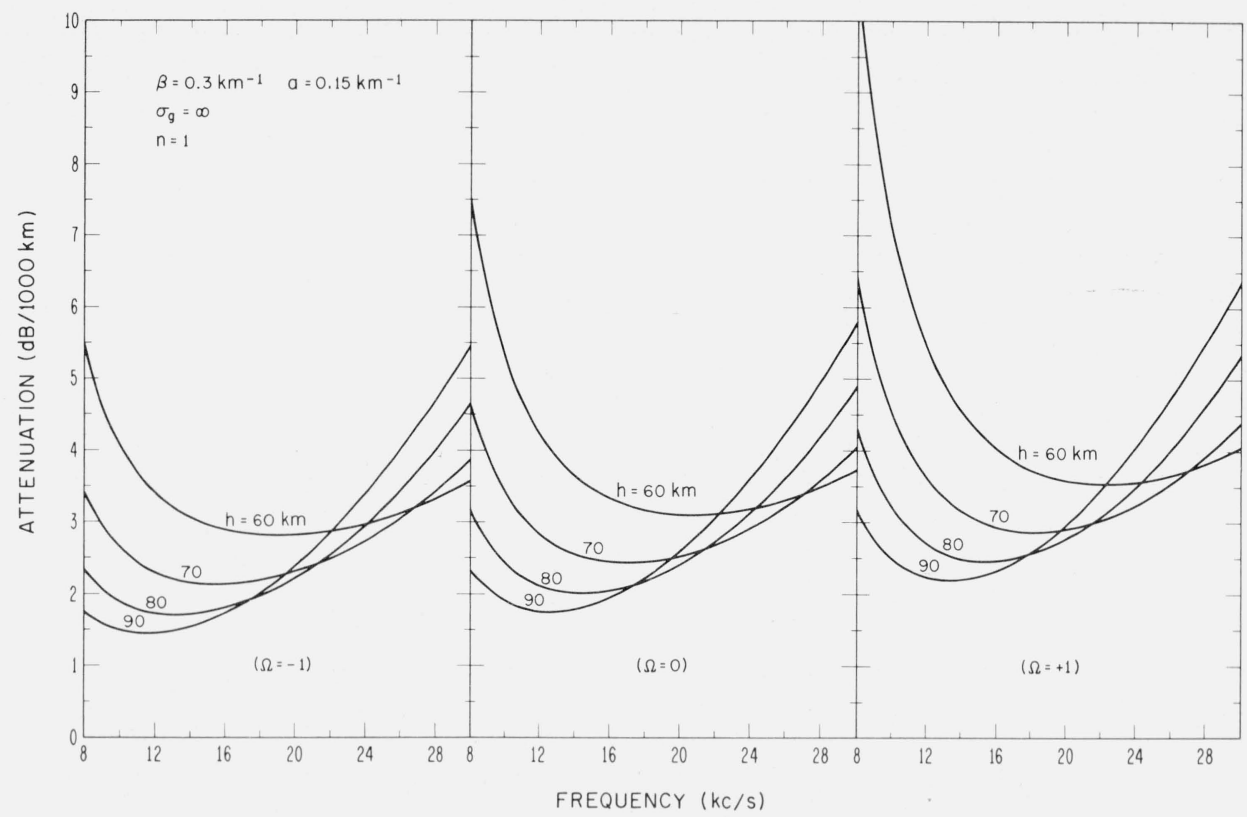

FIGURE 1. Attenuation rate of mode 1 in the earth-ionosphere waveguide for perfectly conducting ground.

[Here, $\Omega<0$ corresponds to propagation from the west towards the east, while $\Omega>0$ corresponds to propagation from the east towards the west.]

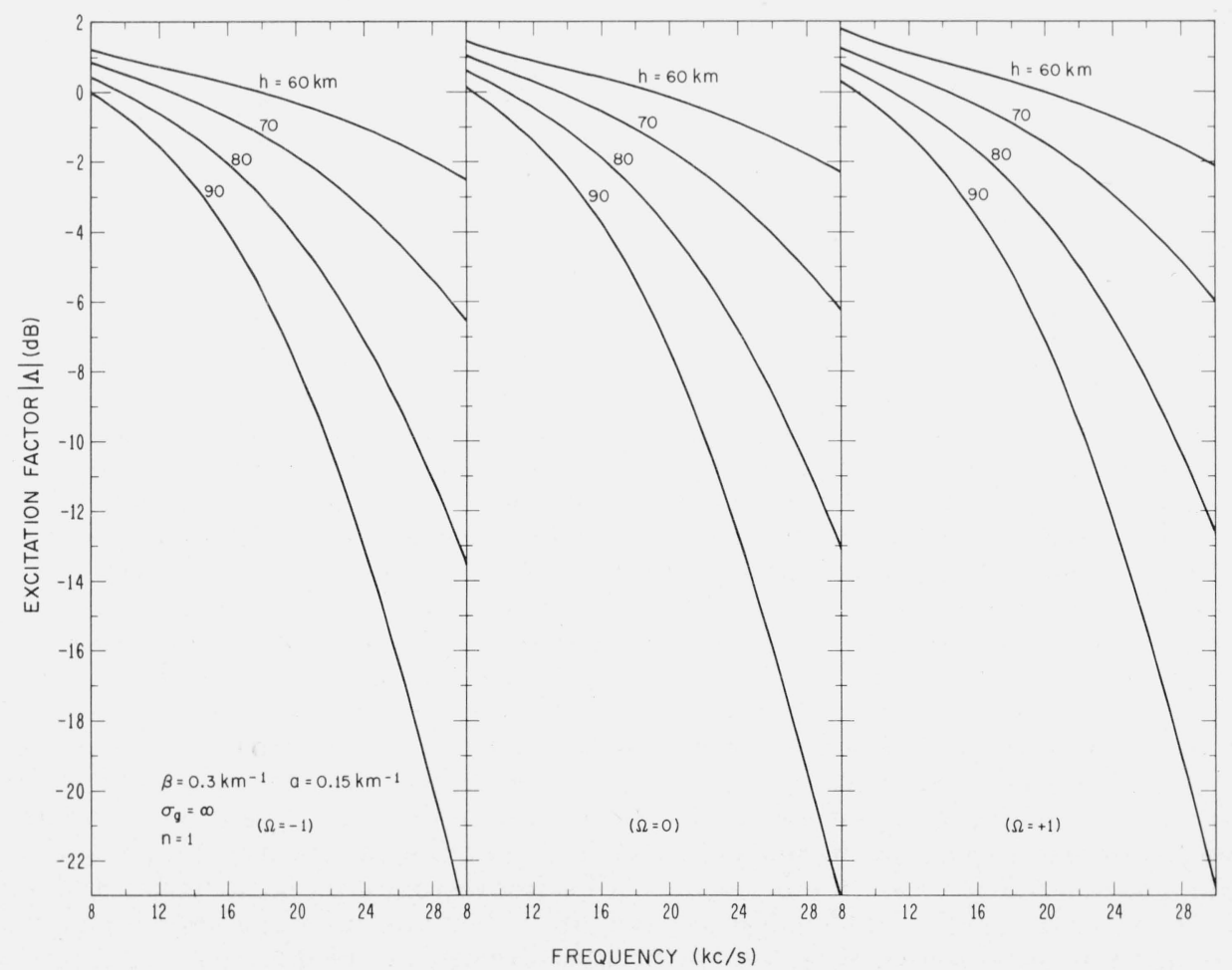

FIGURE 2. The magnitude of the excitation factor of mode 1 in the earth-ionosphere waveguide for perfectly conducting ground. 


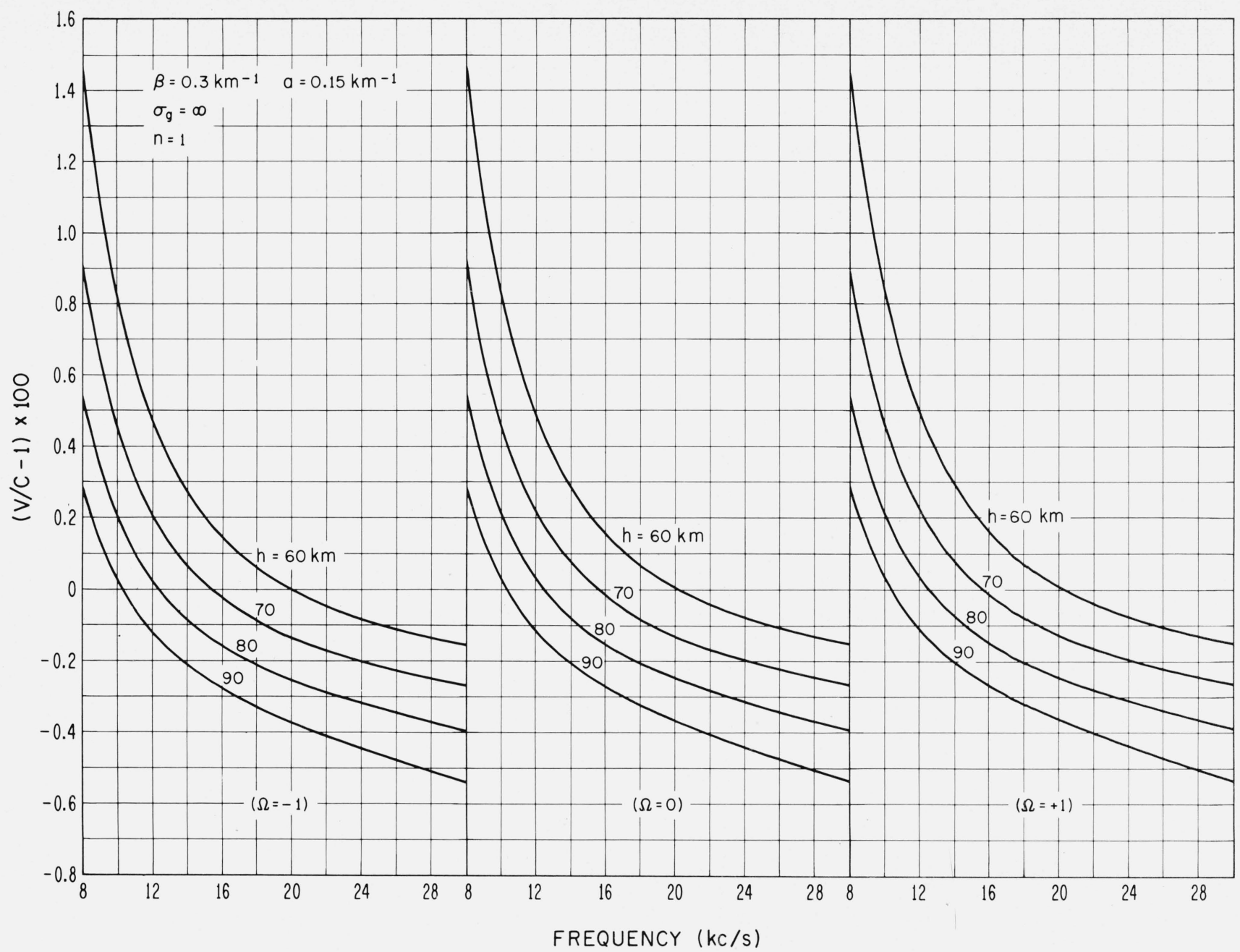

FIGURE 3. Phase velocity of mode 1 in the earth-ionosphere waveguide for perfectly conducting ground.

The phase of the excitation factor for mode 1 is shown in figure 4 which, again, is for the same conditions as figure 1. Generally, these are positive, corresponding to a phase lead. Here, there appears to be some evidence of a nonreciprocal effect since the ordinate values differ for $\Omega=+1$ and $\Omega=-1$.

The attenuation rate for mode 2 is shown in figure 5 for a perfectly conducting ground and the same ionospheric model used in figures 1-4. Again, the nonreciprocal effects are in evidence. Here, it is important to point out that the attenuation rate of mode 2 may be of the same order or even less than mode 1 at the higher end of the VLF band. Consequently, interpretation of experimental data should take this into account. In this paper, no further calculated characteristics of mode 2 for a perfectly conducting ground are shown. However, information of this kind may be obtained in the referenced technical note [W ait and Spies, 1964].

The marked influence of ground conductivity is indicated in figure 6 where the attenuation rate for mode 1 is plotted as a function of frequency for ground conductivities ranging from $\infty$ down to 0.2 millimho $/ \mathrm{m}$. In these curves, the relative dielectric constand $K$ is taken to be 15 . On the basis of these curves, it would appear that ground conductivities less than 1 millimho/m lead to prohibitively high attenuation rates in the daytime (i.e., $h \sim 70 \mathrm{~km}$ ).

The behavior of the excitation factor as a function of frequency, for finitely conducting ground, is illustrated in figure 7 for mode 1 . It is rather interesting to note that $|\Lambda|$ is increased by several $\mathrm{dB}$ as the ground conductivity changes from $\infty$ to 0.2 millimho/m. However, further decreases of ground conductivity, mainly at the higher frequencies, lead to an eventual reduction of the excitation factor.

The interesting curves in figure 8 show that, for a height $h$ of $90 \mathrm{~km}$, the dependence on ground conductivity is relatively weaker (e.g., compare with fig. 6). This is particularly the case at the higher frequencies. From a physical standpoint, this behavior may be attributed to the "earth-detached" character of mode 1 


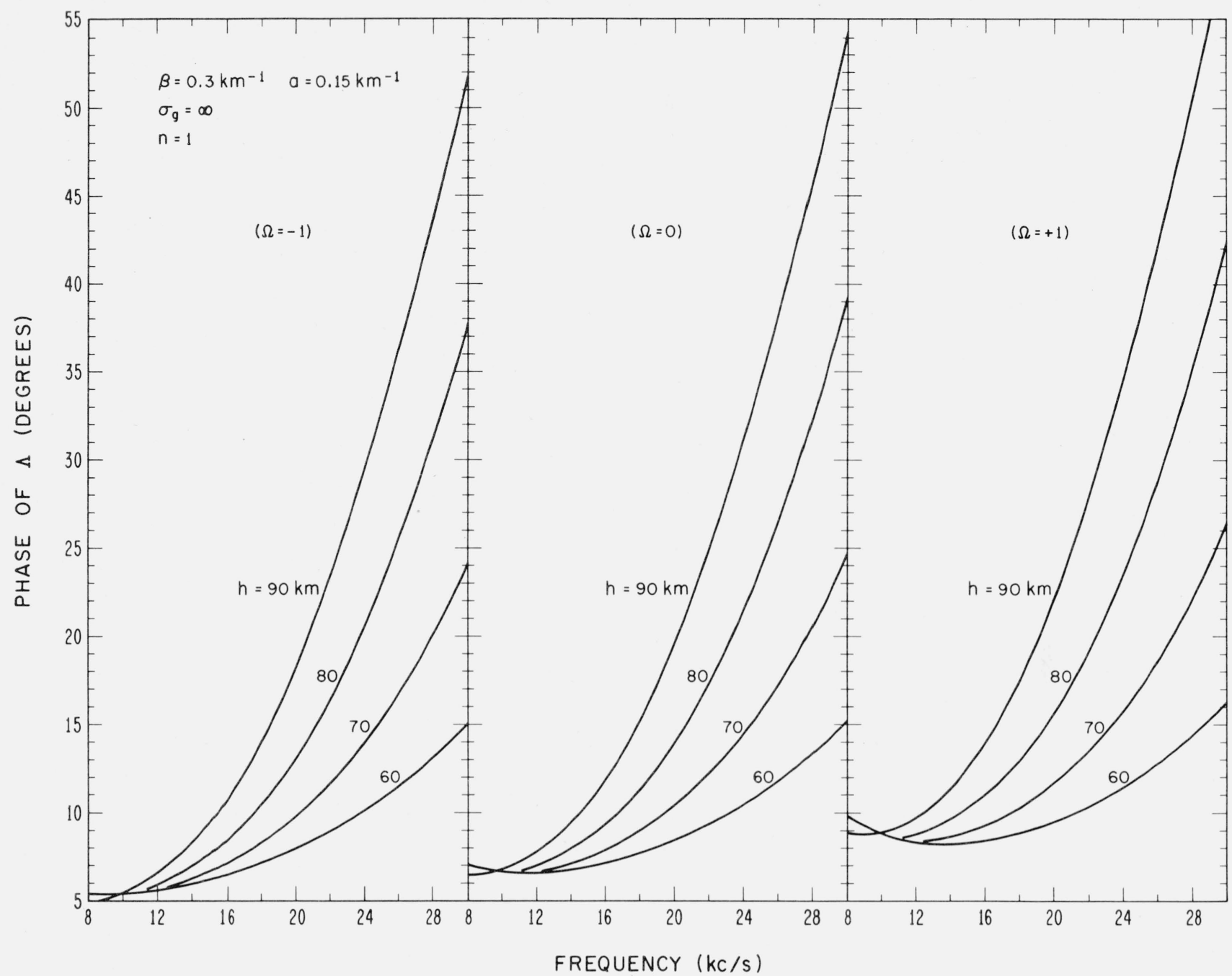

FIGURE 4. Phase of the excitation factor of mode 1 in the earth-ionosphere waveguide for perfectly conducting ground.

in the earth-ionosphere waveguide when the reflecting height is of the order of $90 \mathrm{~km}$ or greater [W ait, 1962]. Thus, in this limiting situation, the energy is being predominantly guided along the lower edge of the ionosphere without being affected appreciably by the presence of the earth.

The attenuation rate and the excitation factor for various ground conductivities are shown in figures 9 and 10 , respectively, for $h=60 \mathrm{~km}$ and $n=1$. Similar curves are shown in figures 11 and 12 for $h=80 \mathrm{~km}$.

A very important feature associated with finite ground conductivity is shown in figure 13 where the attenuation rate is plotted as a function of the ground conductivity for $h=70 \mathrm{~km}$. As indicated, the attenuation for $n=1$ is seen to have a maximum value for a specific value of ground conductivity which itself depends on frequency. In general, the lower frequencies (i.e., around $10 \mathrm{kc} / \mathrm{s}$ ) are characterized by increasingly high attenuation as the ground conductivity is decreased from 10 to $10^{-2}$ millimho/m. However, for the higher frequencies, there is a maximum below which the attenuation rate decreases with further decreases in ground conductivity. This effect is reminiscent of a Brewster angle phenomenon, although the analogy should not be carried too far since geometrical-optical concepts are of questionable validity here. Nevertheless, a peak in the absorption does suggest that the normal wave impedance in the waveguide mode is nearly matched to the impedance of the ground. As indicated in figure 13, the effect is not critically dependent on the relative dielectric constant of the ground.

The attenuation rate curves in figure 14 are similar to those in figure 13 except that now $h=90 \mathrm{~km}$ which might be more typical of nighttime conditions. The same maxima in the attenuation curves for $n=1$ are evident. However, in this case, the ground conductivity dependence is generally weaker, particularly 


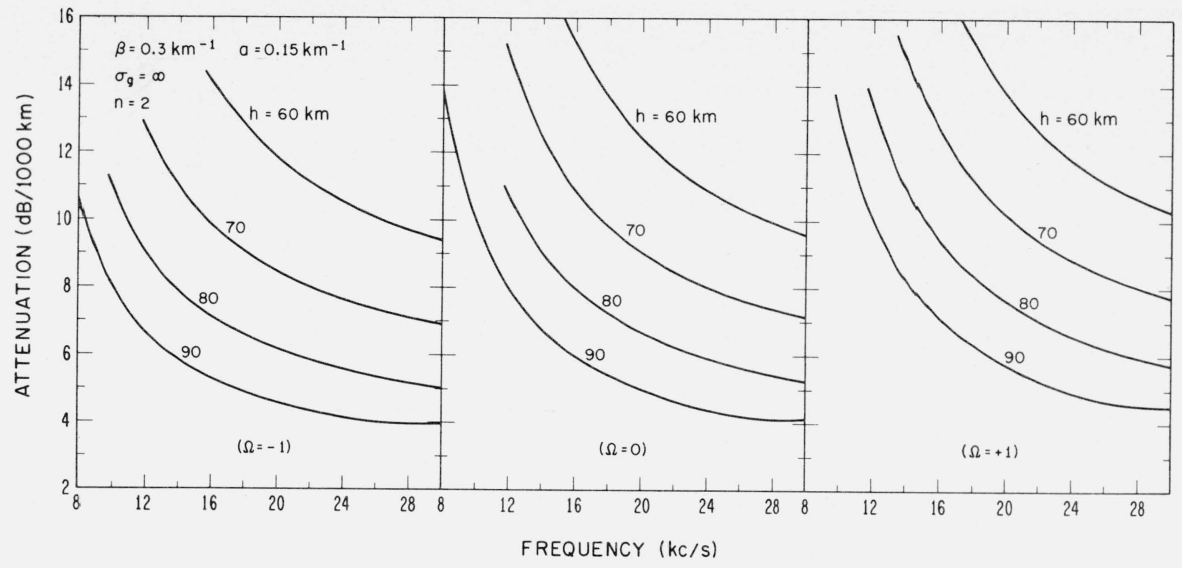

FIGURE 5. Attenuation rate of mode 2 in the earth-ionosphere waveguide for perfectly conducting ground.

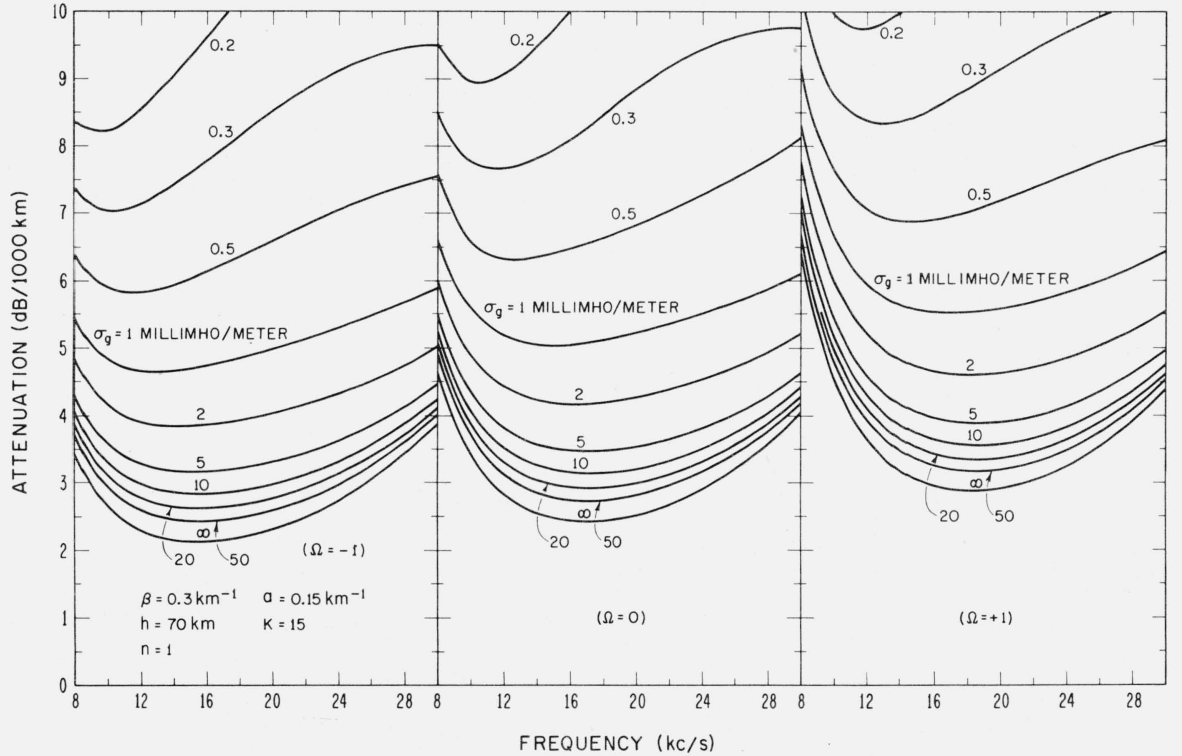

FIGURE 6. Attenuation rate of mode 1 for $\mathrm{h}=70 \mathrm{~km}$ for various ground conductivities.

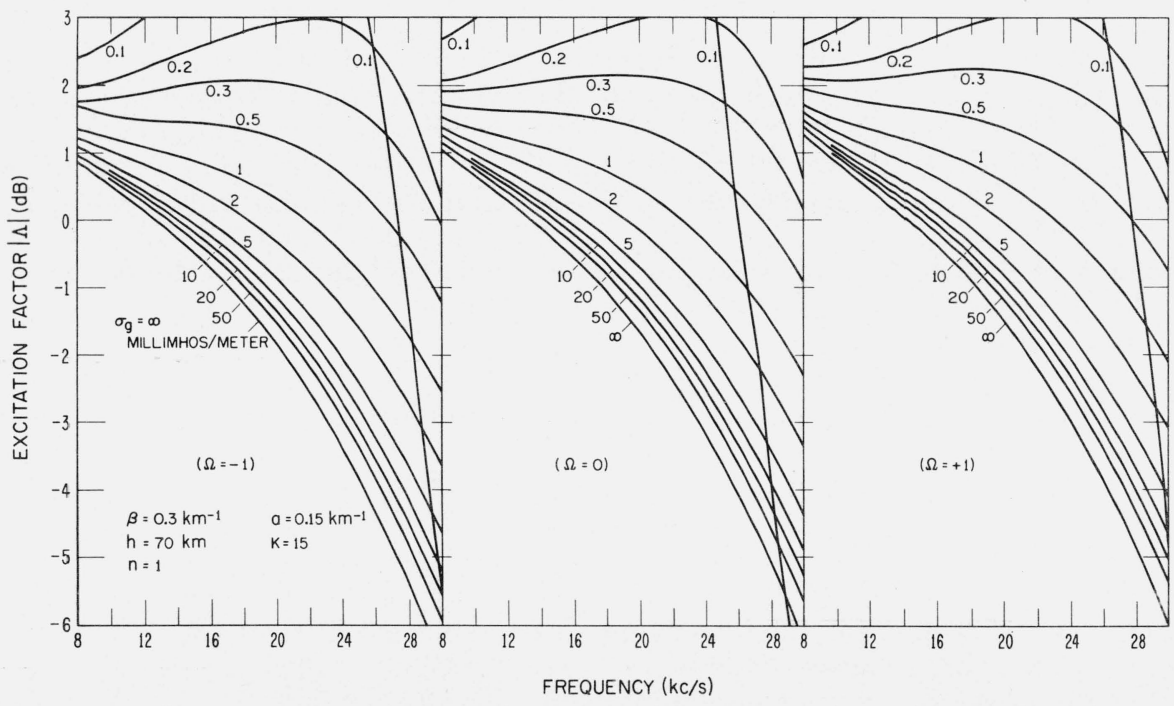

FIGURE 7. Magnitude of excitation factor of mode 1 for $\mathrm{h}=70 \mathrm{~km}$ for various ground conductivities. 


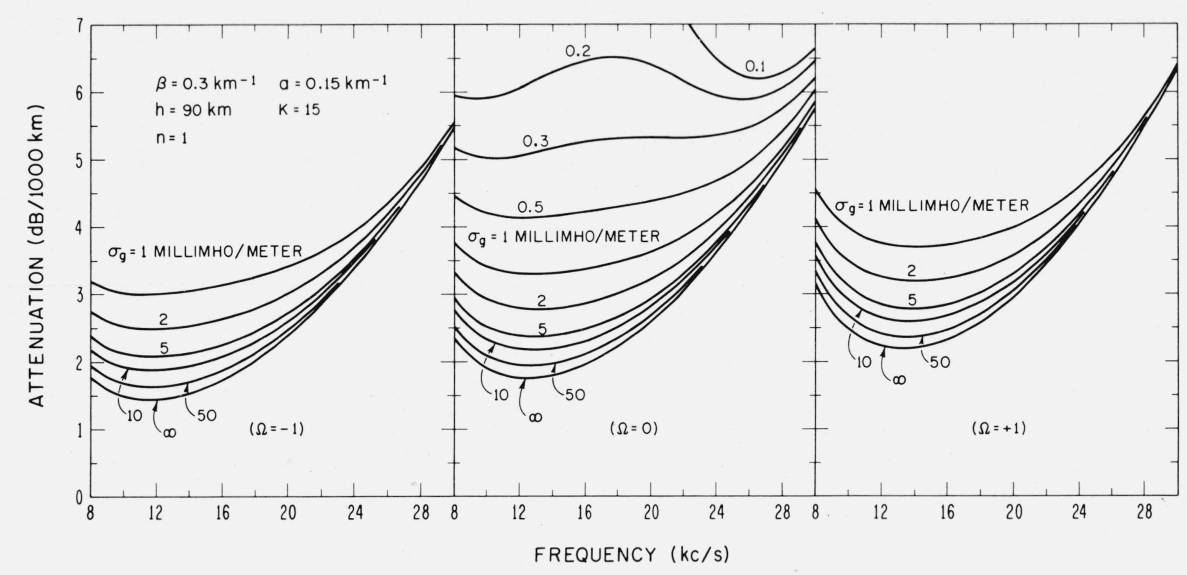

FigURE 8. Attenuation rate of mode 1 for $\mathrm{h}=90 \mathrm{~km}$ for various ground conductivities.

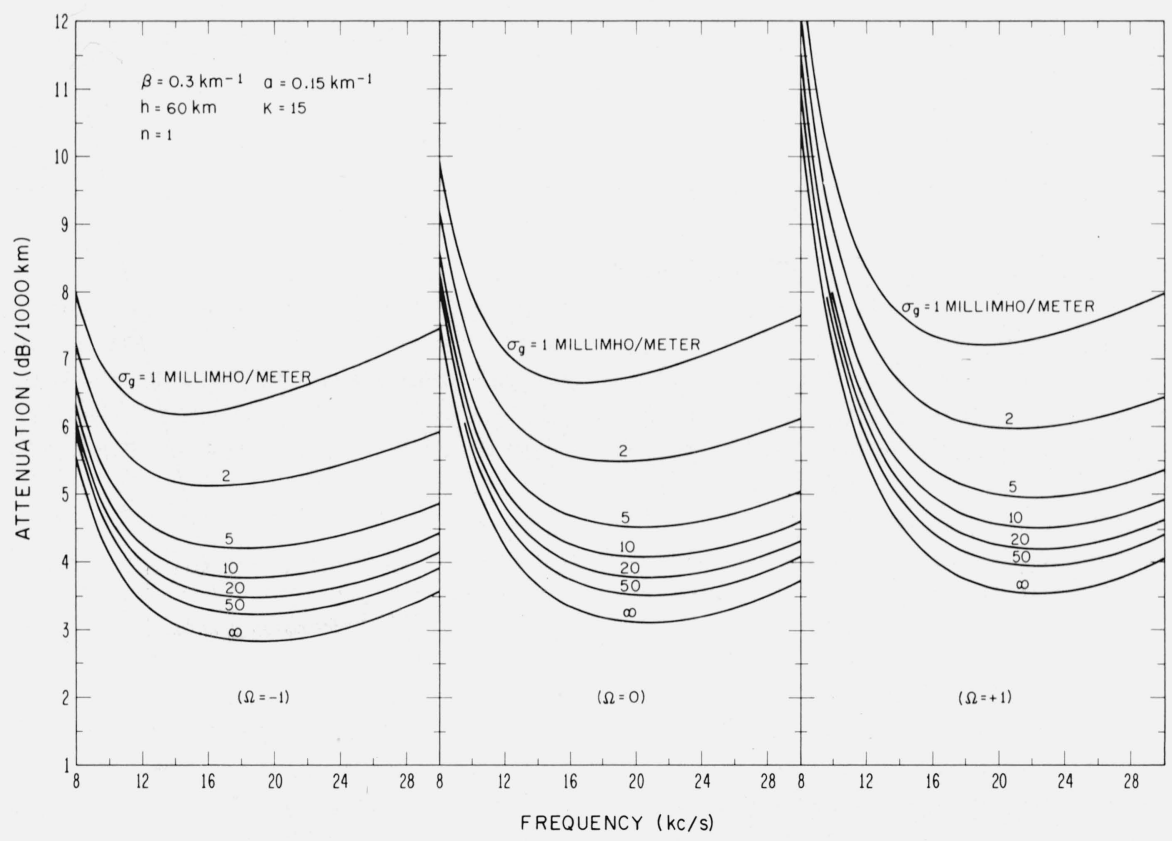

FIGURE 9. Attenuation rate of mode 1 for $\mathrm{h}=60 \mathrm{~km}$ for various ground conductivities.

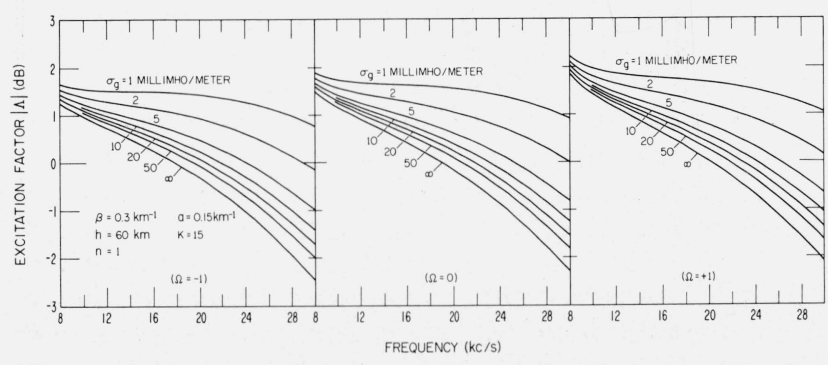

Figure 10. Magnitude of excitation factor of mode 1 for $\mathrm{h}=60 \mathrm{~km}$ for various ground conductivities.

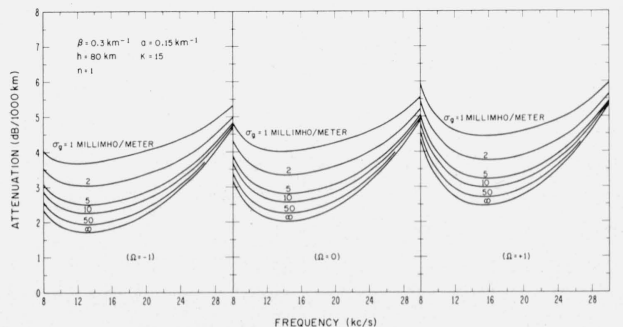

Figure 11. Attenuation rate of mode 1 for $\mathrm{h}=80 \mathrm{~km}$ for various ground conductivities. 


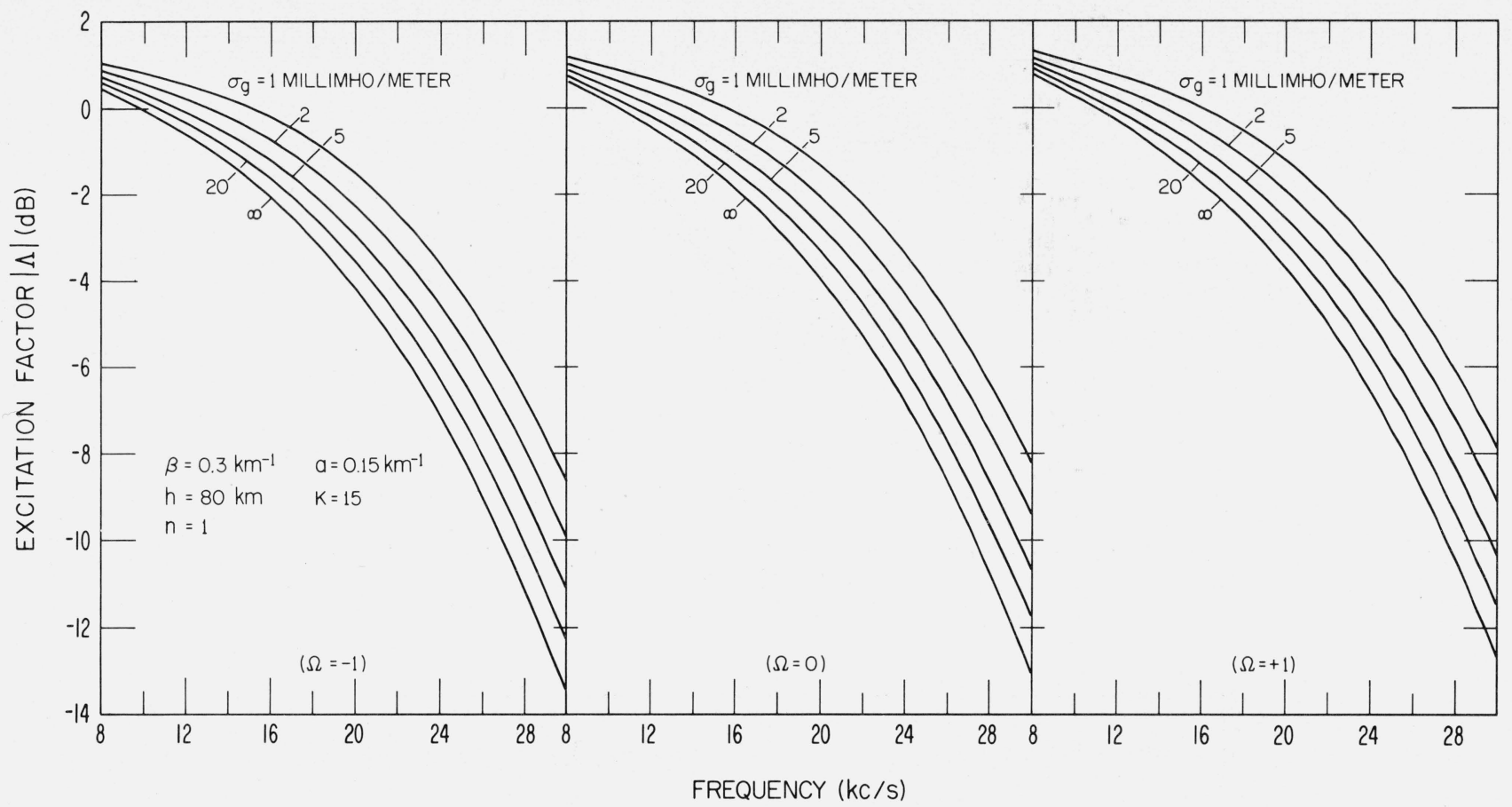

FIGURE 12. Magnitude of excitation factor of mode 1 for $\mathrm{h}=80 \mathrm{~km}$ for various ground conductivities.

at the higher frequencies. In the latter case, the modes have the earth-detached character which is consistent with this behavior.

A number of interesting features are shown in figure 15 where the frequency is $30 \mathrm{kc} / \mathrm{s}$. Here, the attenuation rate and the excitation factors for modes $n=1$ and $n=2$ are plotted as a function of ground conductivity for two anisotropic ionospheric models with $h=90 \mathrm{~km}$. As expected, the attenuation rate for $n=1$ shows only a weak dependence on ground conductivity. On the other hand, the ground conductivity dependence for $n=2$ is very strong and there is a pronounced maximum in the attenuation for a conductivity of about 0.12 millimho/m. For both modes 1 and 2, the excitation factor shows a maximum which tends, to some extent, to offset the increased attenuation rates. It is also clearly evident, from figure 15 , that the earth's magnetic field plays a relatively minor role here.

The relative influences of frequency, earth's magnetic field, and ground conductivity are shown rather clearly in figure 16 where $h=70 \mathrm{~km}$. Consistently, the attenuation rate is least for propagation from west to east (i.e., $\Omega<0$ ) even for relatively poor ground conductivities. However, it may be important to note that the frequency of minimum attenuation is significantly lowered as the ground conductivity is decreased from $\sigma_{g}=\infty$. Of course, this effect is also in evidence in figure 6.

Curves of the excitation factor, are shown in figure 17, corresponding to the conditions of figure 16. As indicated, the ground conductivity has a relatively greater effect than that due to the earth's magnetic field.
The attenuation rate for a fairly wide range of ground conductivities and relative dielectric constants is shown in figure 18. The shape of the attenuation versus frequency curve changes significantly for verylow ground conductivities. Also, in this region, the dependence on the relative dielectric constant of the ground is appreciable.

Curves of the excitation factor, corresponding to the conditions of figure 18, are shown in figure 19. The eventual decrease of the excitation factor for very-low ground conductivities is certainly evident here.

The rather interesting behavior of the phase velocity for low ground conductivities is seen in figure 20 . In general, a decrease of ground conductivity is associated with a reduced phase velocity. However, in certain extreme cases, the reverse seems to be the case. Furthermore, here, the dispersion curve has a negative slope which suggests that the waves are "backward" in the sense that phase and energy flow in opposing directions. However, the physical significance of this phenomenon is questionable since the associated attenuation rates are prohibitively high.

The phase of the excitation factor, corresponding to the conditions in figures 18,19 , and 20 , is shown in figure 21 . It is certainly evident here that the lowground conductivity leads to a substantial phase lag.

Finally, the effect of the relative dielectric constant on the frequency dependence of the attenuation is shown in figure 22 for a relatively poor ground conductivity. The effect is really not very important, although it is interesting to note that the larger dielectric constants lead to lower attenuations at higher frequencies for $n=1$, while the effect is reversed at lower frequencies. 


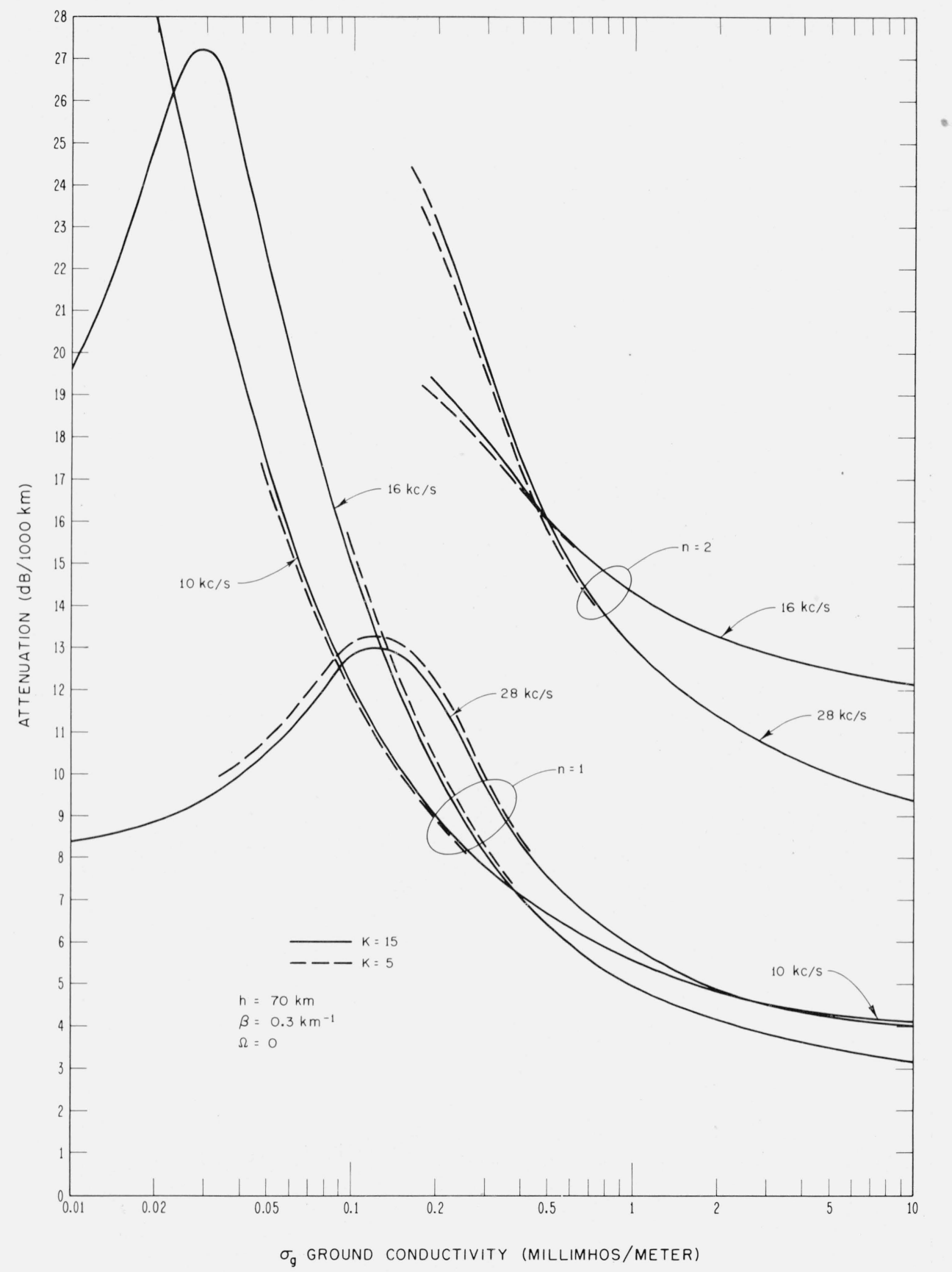

FigURE 13. Attenuation rate for $\mathrm{h}=70 \mathrm{~km}$ as a function of ground conductivity for several frequencies.

\section{Concluding Remarks}

The results given in this paper should be of some use in making estimates of the importance of poorly conducting ground in the long-distance propagation of VLF radio waves. However, it should be stressed that the model used is highly idealized in that the ground is assumed to be homogeneous throughout the length of the path. For propagation over mixed paths, the situation may be much more complicated since discontinuities such as a land/sea boundary will cause conversion of modes from one order to another [e.g., see Wait, 1965]. However, the fact remains that poorly conducting portions of the actual path will lead to considerable weakening of the received signal strength.

The authors thank William E. Garner of the U.S. Naval Research Laboratory, who suggested that these calculations be carried out. 


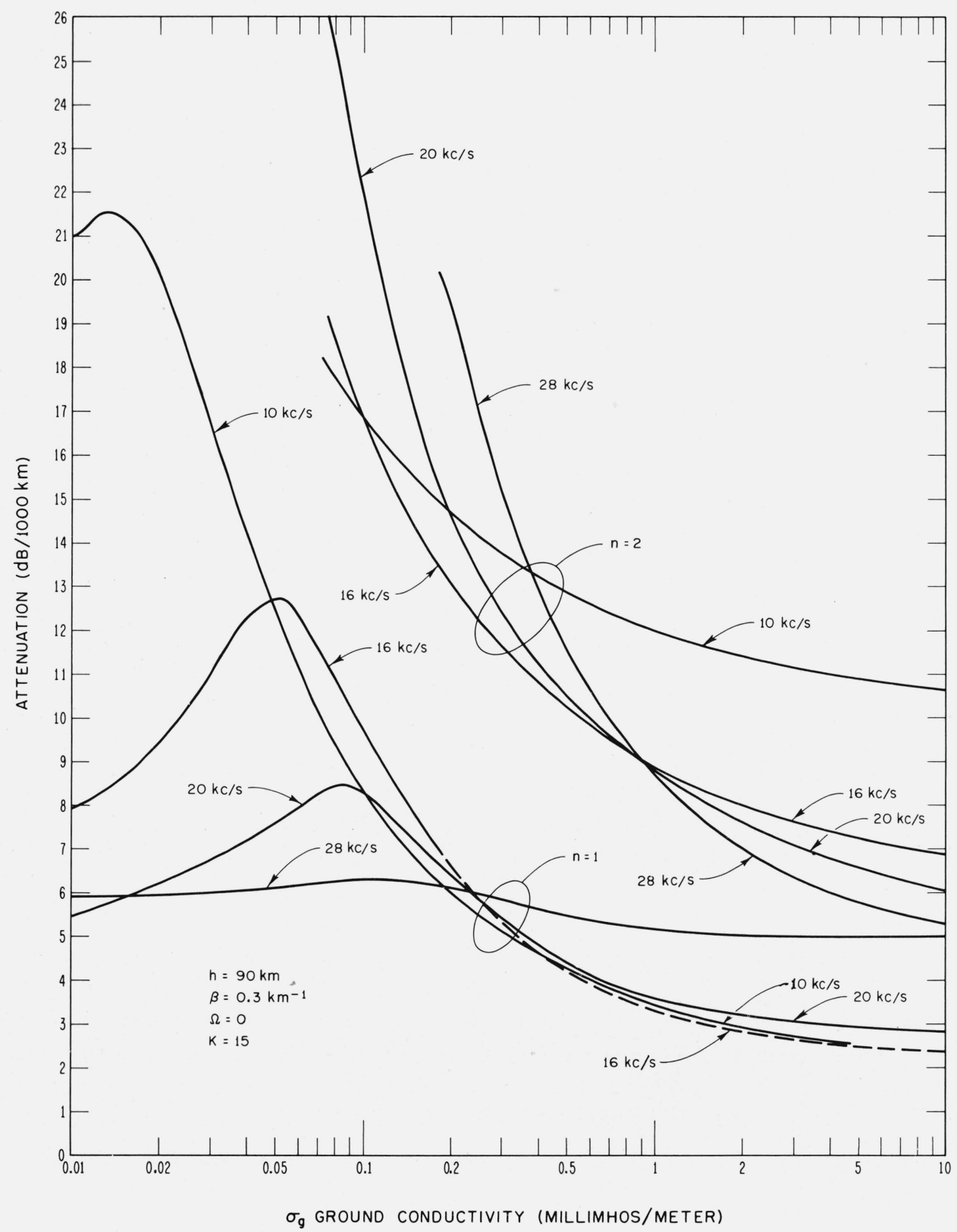

FIGURE 14. Attenuation rate for $\mathrm{h}=90 \mathrm{~km}$ as a function of ground conductivity for several frequencies. 


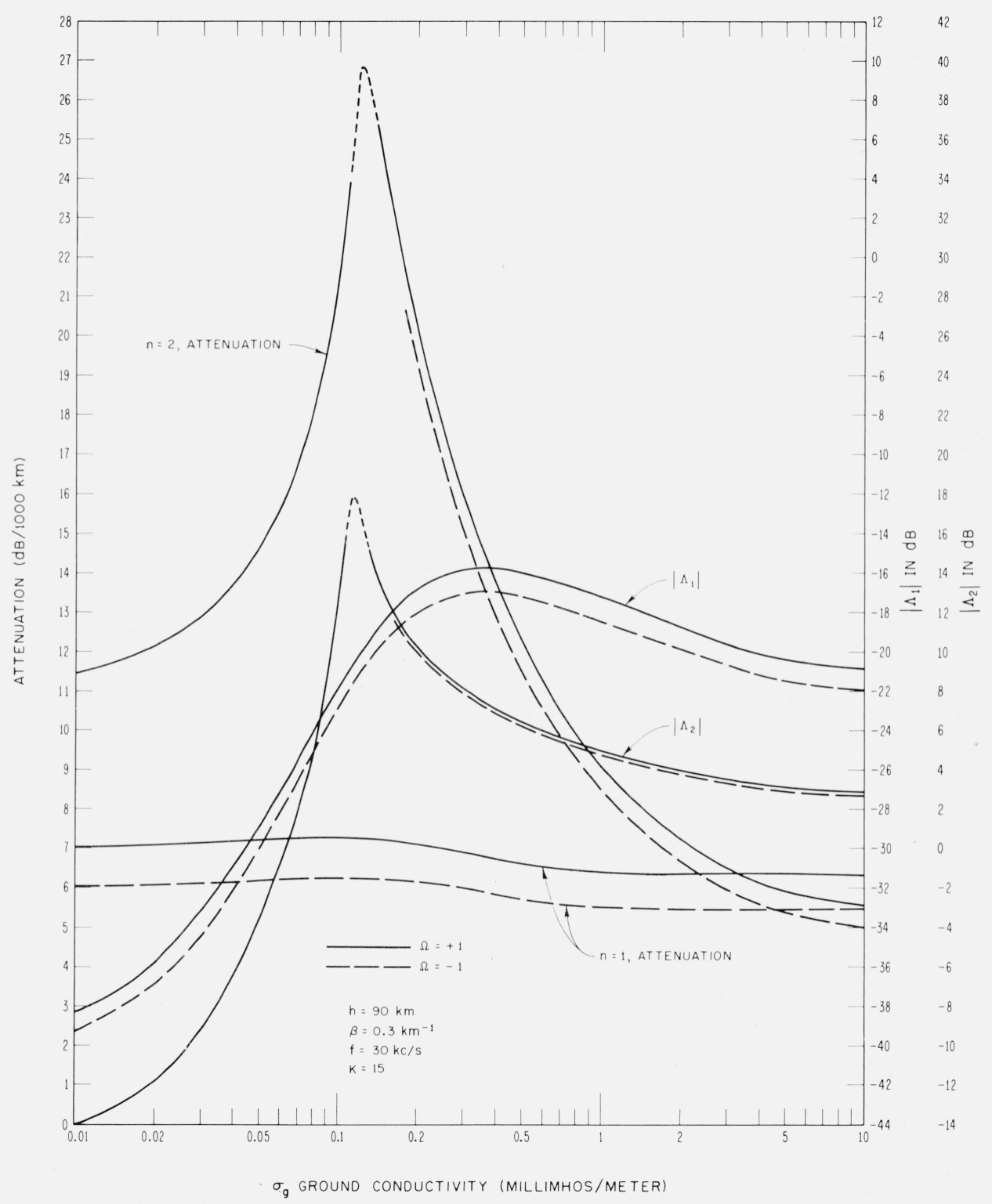

FIGURE 15. Attenuation rate as a function of ground conductivity showing nonreciprocal effect of the earth's magnetic field. 


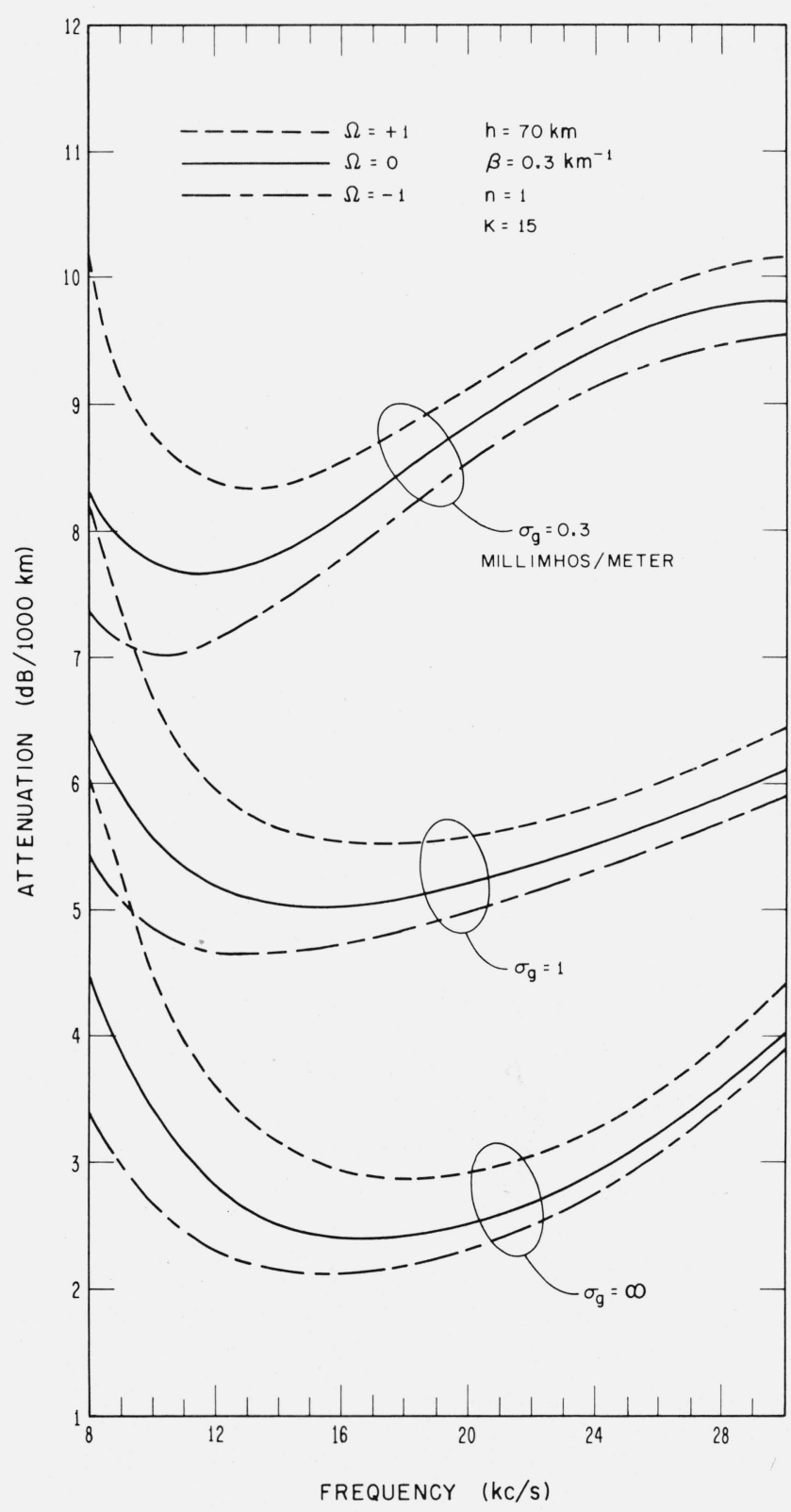

FIGURE 16. Attenuation rate showing relative influence of earth's magnetic field and ground conductivity.

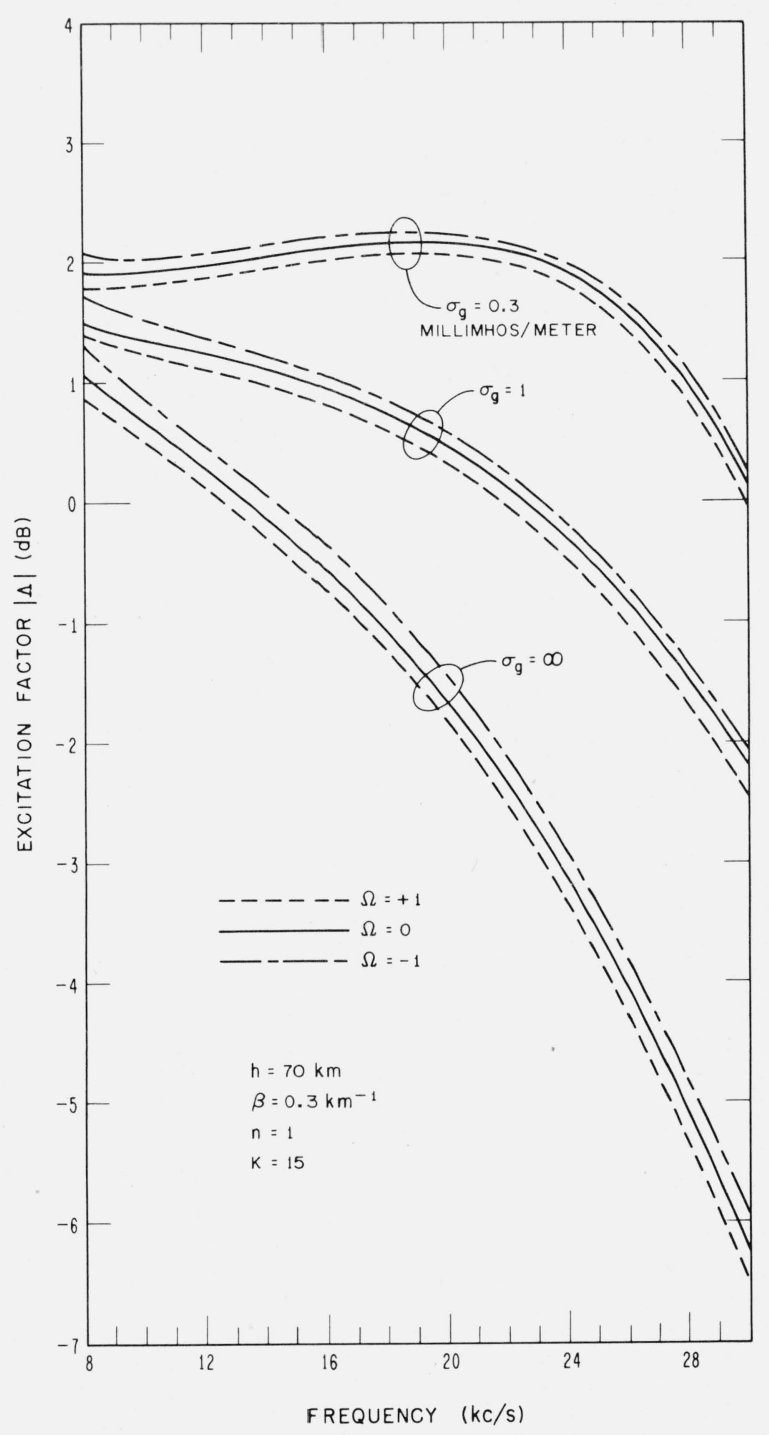

FIGURE 17. Magnitude of excitation factor showing relative infuence of earth's magnetic field and ground conductivity. 


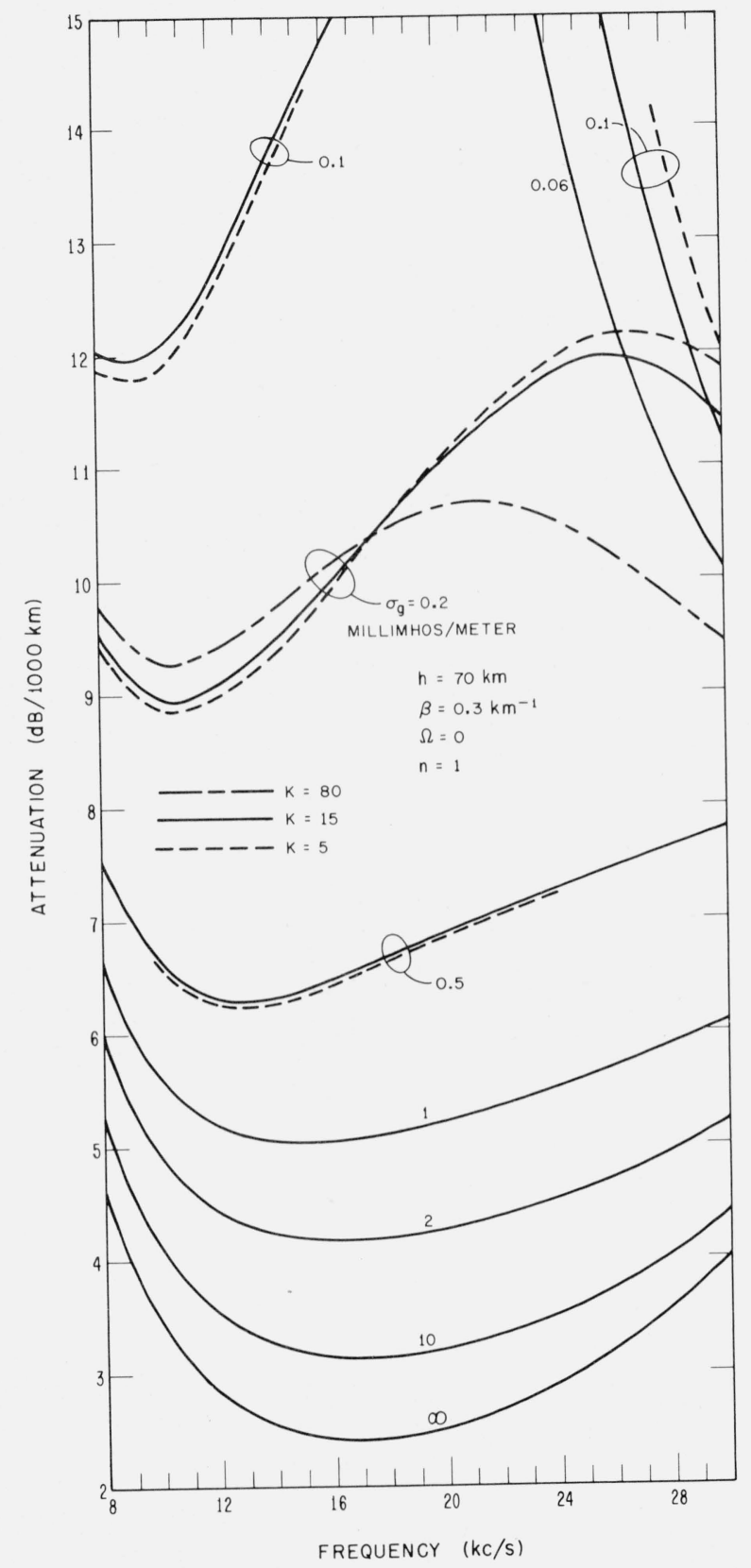

Figure 18. Attenuation rate for a wide range of ground conductivity showing effect of dielectric constant changes.

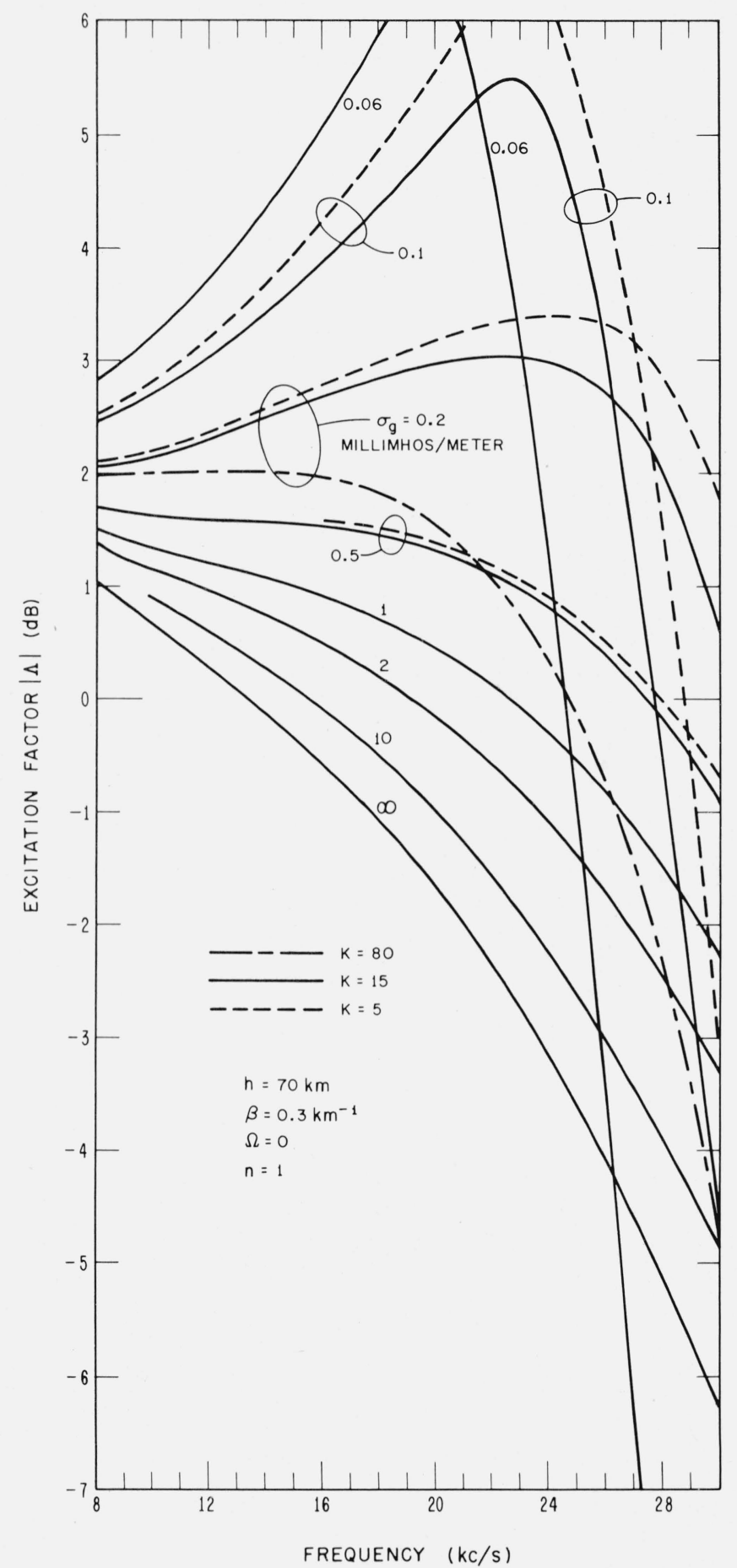

Figure 19. Magnitude of the excitation factor for a wide range of ground conductivity showing effect of dielectric constant changes. 

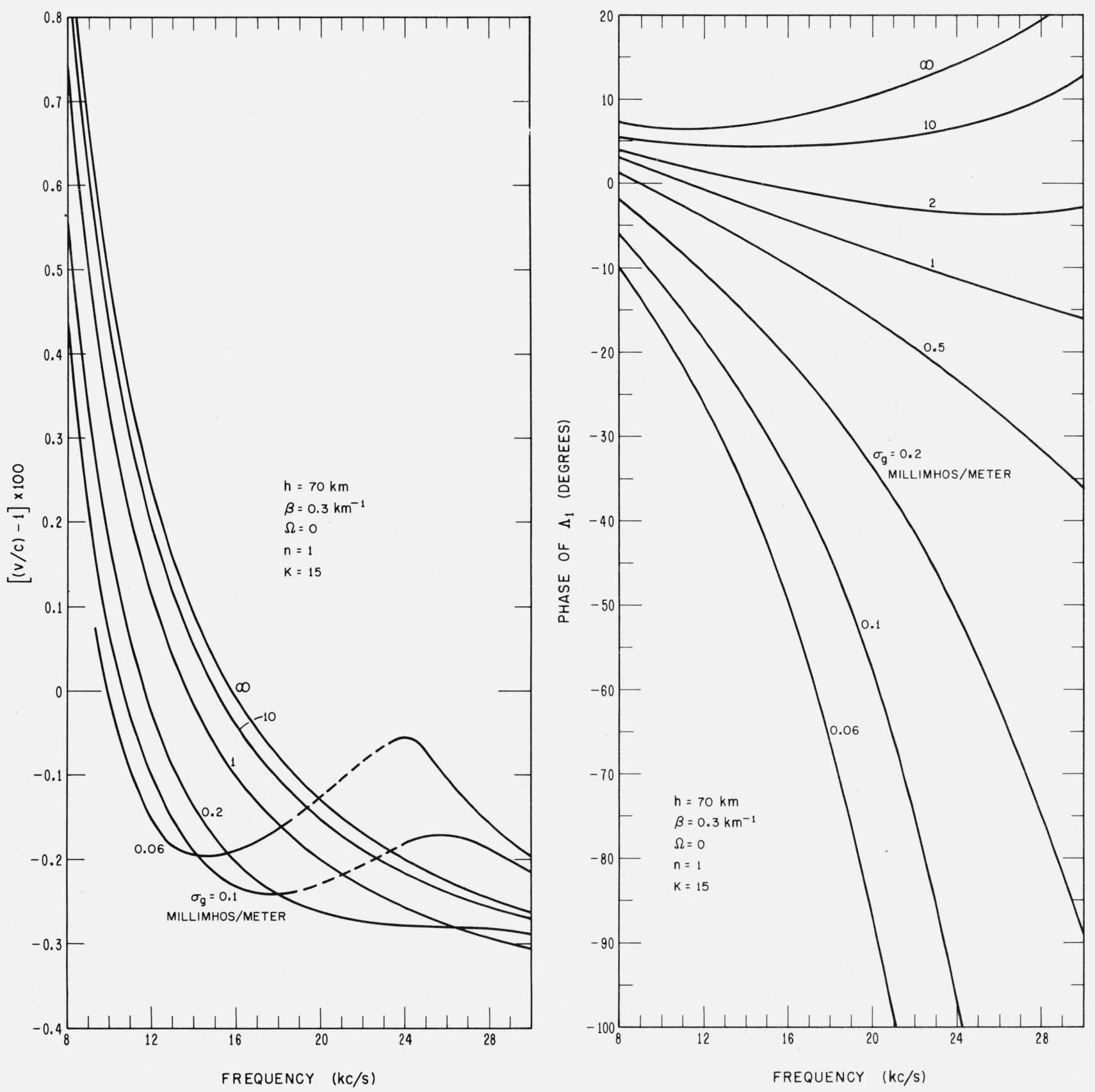

FIGURE 20. Phase velocity for a wide range of ground conductivities.

FIGURE 21. Phase of excitation factors for a wide range of ground conductivities. 


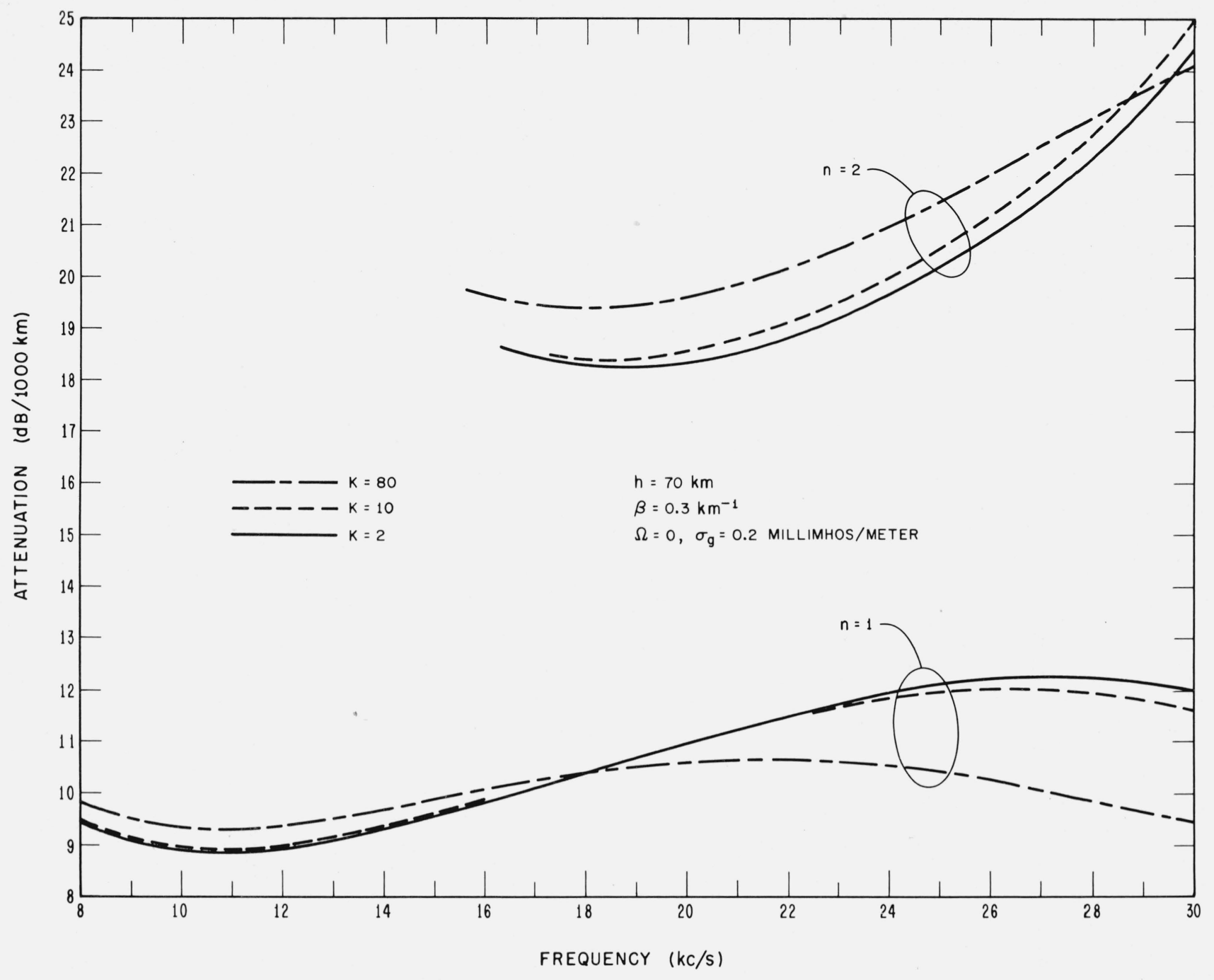

FIGURE 22. Attenuation rate showing importance of dielectric constants for a low ground conductivity.

\section{References}

Al'pert, Ya. L. (1960), Radio wave propagation and the ionosphere (Academy of Sciences Press, Moscow, USSR).

Budden, K. G. (1962), The waveguide mode theory of wave propagation (Prentice-Hall, Englewood Cliffs, N.J.)

Crombie, D. D. (1958), Differences between east-west and westeast propagation of VLF signals over long distances, J. Atmospheric Terrest. Phys. 12, 110-117.

Galejs. J. (May 1965), On the terrestrial propagation of ELF and VLF waves in the presence of a radial magnetic field, Radio Sci. J. Res. NBS 69D, No. 5, 705-720.

Johler, J. R., and L. A. Berry (Nov.-Dec. 1962), Propagation of terrestrial radio waves of long wavelengths, J. Res, NBS 66D, (Radio Prop.), No. 6, 737-762.

Obayashi, T., S. Fujii, and T. Kodokoro (1959), An experimental proof of the mode theory of ionospheric propagation, J. Geomag. Geoelec. Japan 10, 47-55.

Taylor, W. L. (July-Aug. 1960), Daytime attenuation rates in the VLF band using atmospherics, J. Res. NBS 64D (Radio Prop.), No. 4, 349-355.

Volland, H. (July-Aug. 1961), Comparison between mode theory and ray theory for VLF propagation, J. Res. NBS 65D (Radio Prop.), No. 4, 357-361.

Wait, J. R. (June 1957), The mode theory of VLF ionospheric propagation for finite ground conductivity, Proc. IRE 45, No. 6, 760-767.
Wait, J. R. (1962), Electromagnetic waves in stratified media ch. 7, (Pergamon Press, Oxford).

Wait, J. R. (July 1965), Influence of an inhomogeneous ground on the propagation of VLF radio waves in the earth-ionosphere waveguide, Radio Sci. J. Res. NBS 69D, No. 7, 969-975.

Wait, J. R., and K. P. Spies (Dec. 30, 1964), Characteristics of the earth-ionosphere waveguide for VLF radio waves, NBS Tech. Note No. 300. [For sale by the Supt. of Documents, U.S. Gov't. Printing Office, Washington, D.C. 20402, 50 cents.] A numerical supplement to this technical note is available on request from the authors.

Watt, A. D., and R. D. Croghan (Jan. 1964), Comparison of observed VLF attenuation rates and excitation factors with theory, Radio Sci. J. Res. NBS 68D, No. 1, 1-9.

\section{Additional References}

Bremmer, H. (1958), Propagation of electromagnetic waves, Handbuch der Physik 16, 243.

Crombie, D. D., and A. G. Jean (May 1964), The guided propagation of ELF and VLF radio waves between the earth and the ionosphere, J. Res. NBS 68D, No. 5, 584-588.

Johler, J. R., and L. A. Berry (1 Oct. 1964), A complete mode sum for LF, VLF, ELF terrestrial radio wave fields, NBS Mon. No. 78.

Rayleigh, Lord J. W. Strutt (1910), The problem of the whispering gallery, Phil. Mag. 20, 1001-1004.

(Paper 69D10-570) 